\title{
VALUE REGIONS FOR CONTINUED FRACTIONS ${ }^{1}$
}

\author{
W. T. SCOTT AND H. S. WALL
}

1. Introduction. In a recent paper ${ }^{2}$ we showed that if $a_{2}, a_{3}, a_{4}, \cdots$ lie in or upon the parabola

$$
|z|-\Re(z)=\frac{1}{2}
$$

then the continued fraction

$$
\frac{1}{1}+\frac{a_{2}}{1}+\frac{a_{3}}{1}+\frac{a_{4}}{1}+\cdots
$$

converges if and only if the series $\sum\left|b_{n}\right|$ diverges, where $b_{1}=1$, $a_{n}=1 / b_{n-1} b_{n},(n=2,3,4, \cdots)$, the series being considered as divergent if some $a_{n}$ vanishes. Further, if $z$ lies outside this parabola, the periodic continued fraction

$$
\frac{1}{1}+\frac{\bar{z}}{1}+\frac{z}{1}+\frac{\bar{z}}{1}+\frac{z}{1}+\cdots,
$$

in which $\bar{z}$ is the conjugate of $z$, diverges, so that the parabola (1.1) is the "best" curve symmetrical with respect to the real axis.

The principal object of the present paper is to show that when $a_{2}, a_{3}, a_{4}, \ldots$ lie in or upon the parabola (1.1) then all the approximants of (1.2) lie in or upon the circle

$$
|z-1|=1 \text {. }
$$

If $z_{1}$ is any value of $z$ not zero which is in or upon this circle, then there is a value $z$ in or upon the parabola (1.1) such that the value of the continued fraction (1.3) is $z_{1}$, and therefore the circular domain is the "best" domain.

2. Fundamental lemma. If we adopt the notation

then

$$
v_{n}=\frac{1}{1}+\frac{a_{n+1}}{1}+\frac{a_{n+2}}{1}+\cdots
$$

$$
v_{n}=\frac{1}{1+a_{n+1} v_{n+1}}, \quad n=1,2,3, \cdots .
$$

${ }^{1}$ Presented to the Society under the title $A$ geometrical method in the theory of continued fractions, November 22, 1940.

${ }^{2}$ W. T. Scott and H. S. Wall, A converge theorem for continued fractions, Transactions of this Society, vol. 47 (1940), pp. 155-172, p. 166. We refer to this paper later as CT. 
Thus the continued fraction (1.2) may be regarded as a succession of linear transformations of elements $v_{n}$ belonging to a set $V$, where the transformations depend upon elements $a_{n}$ of a set $U$. This notion leads us to formulate the following lemma.

Lemma. If there exist in the complex plane sets of points $U$ and $V$ such that

(i) $1 /(1+u) \in V$ when $u \in U$,

(ii) $1 /(1+u v) \in V$ when $u \in U, v \in V$,

then every approximant of the continued fraction (1.2), beginning with the second, lies in $V$ when the elements $a_{2}, a_{3}, a_{4}, \cdots$ lie in $U$.

Let $A_{n} / B_{n}$ denote the $n$th approximant of (1.2). Then, by (i), $A_{2} / B_{2}=1 /\left(1+a_{2}\right) \in V$ when $a_{2} \in U$. Suppose now that we have verified that $A_{n} / B_{n} \in V$ when the $a_{n}$ 's are in $U$. Then

$$
A_{n+1} / B_{n+1}=1 /\left(1+a_{2} v\right),
$$

where $v=1 / 1+a_{3} / 1+a_{4} / 1+\cdots+a_{n+1} / 1$, and is in $V$ if $a_{3}, a_{4}, \cdots, a_{n+1}$ are in $U$ by our assumption. Hence, by (ii), $A_{n+1} / B_{n+1}$ is in $V$ when $a_{2}, a_{3}, \cdots, a_{n+1}$ are in $U$. This proves the lemma by induction.

We shall call the set $U$ an element region for the continued fraction (1.2). As $a_{2}, a_{3}, a_{4}, \cdots$ range over $U$, the values of the approximants of (1.2), and the values of (1.2), when convergent, constitute a set $V$ which we call the value region corresponding to the element region $U$. These sets $U$ and $V$ satisfy the conditions of the lemma.

The element region $U$ is called a convergence region for (1.2) if this continued fraction remains convergent when the $a_{n}$ 's vary independently over $U$. A convergence region is necessarily bounded, for otherwise the $a_{n}$ 's could be so chosen that the series $\sum\left|b_{n}\right|$ would converge, which implies divergence of the continued fraction by oscillation.

In the next section we shall obtain the value region for (1.2) when the element region is the set of points in and upon the parabola given by (1.1).

3. The parabola-circle theorem. If $a_{2}, a_{3}, a_{4}, \cdots$ lie within or upon the parabola (1.1) then all the approximants of the continued fraction (1.2) lie within or upon the circle $|z-1|=1$. This circular region with the point $z=0$ removed is the value region corresponding to the parabolic element region.

We seek first a region $V$, not necessarily the value region, corresponding to the parabolic element region $U$, such that $U$ and $V$ satisfy the conditions of the lemma of $\S 2$. If $|z|-\Re(z) \leqq \frac{1}{2}$, the continued fraction (1.3) converges to the value 


$$
w=\frac{-1+2 i y+\left(4 x+1-4 y^{2}\right)^{1 / 2}}{2(x+i y)}
$$

where $z=x+i y$. If $w=\xi+i \eta$, then

$$
\xi=\frac{1}{2 x+1}, \quad \eta=\frac{2 y}{2 x+1},
$$

when $|z|-\Re(z)=\frac{1}{2}$. Thus $w$ traverses the circle $|w-1|=1$ as $z$ traverses the parabola $|z|-\Re(z)=\frac{1}{2}$. From the nature of this mapping of the parabola into the circle it is clear that as $z$ ranges over the interior of the parabola, $w$ ranges over the whole interior of the circle. The value $w=0$ is not assumed for a finite value of $z$. These considerations show that the region $V$ which we seek must contain the region

$$
|z-1| \leqq 1, \quad z \neq 0 .
$$

We shall show that the conditions of the lemma are satisfied when $U$ is the parabolic region and $V$ is the region (3.2).

If $u \in U, v \in V, u=\xi+i \eta, v=x+i y$ we have

$$
(x-1)^{2}+y^{2} \leqq 1, \quad \eta^{2} \leqq \xi+\frac{1}{4} .
$$

Let $v_{1}=1 /(1+u v)=p+i q$. Then the condition that $v_{1}$ shall lie in $V$, namely: $(p-1)^{2}+q^{2} \leqq 1$, is seen to reduce to the condition

$$
x \xi-y \eta \geqq-\frac{1}{2} .
$$

The form of this inequality suggests that we examine the polars of the point $\xi+i \eta$ relative to the hyperbola $x^{2}-y^{2}=-\frac{1}{2}$ when $\eta^{2}=\xi+\frac{1}{4}$. These polars form the one-parameter family of lines

$$
x\left(\eta^{2}-\frac{1}{4}\right)-y \eta=-\frac{1}{2}, \quad-\infty<\eta<+\infty .
$$

The envelope of this family is the circle $(x-1)^{2}+y^{2}=1$. Thus the line $x \xi-y \eta=-\frac{1}{2}$ is tangent to the circle if $\xi+i \eta$ is on the parabola. One can easily see that if $\xi+i \eta$ is inside the parabola this line does not cut the circle. Hence if $\xi+i \eta$ is in the parabola, and $x+i y$ in the circle, the points $x+i y$ and the origin lie on one side of this line. It follows that the inequality (3.3) holds.

We have proved that $U$ and $V$, as defined, satisfy the condition (ii) of the lemma. Since $v=1$ is in $V$, it follows that (i) holds.

From the way in which we arrived at (3.2) as the locus of values of the continued fraction (1.3) as $z$ ranges over the parabola, and from the observation that the value 0 cannot be assumed by (1.2) or any of its approximants when the $a_{n}$ 's lie in the parabola, we now con- 
clude that (3.2) is the value region corresponding to the parabolic element region. Our parabola-circle theorem is now completely proved.

It is interesting to note that the point of tangency of the line $x \xi-y \eta=-\frac{1}{2}$ to the circle $(x-1)^{2}+y^{2}=1$ when $\xi+i \eta$ is on the parabola is given by

$$
x=1 /(2 \xi+1), \quad y=2 \eta /(2 \xi+1) .
$$

Hence we have again the projective mapping (3.1).

4. Circular bounds for the value of a continued fraction. Instead of allowing $a_{2}, a_{3}, a_{4}, \cdots$ to vary independently over the element region $U$, one may suppose certain of the $a_{n}$ 's fixed in $U$ and inquire as to the region $V$ in which the value of the continued fraction must lie when it converges. In this way one may, for example, obtain an estimate for the error committed in using a certain approximant instead of the value of the continued fraction. We shall prove the following theorem.

THEOREM 4.1. If $a_{2}, a_{3}, a_{4}, \cdots$ lie within or upon the parabola (1.1), then the sequence of even approximants of (1.2), which is necessarily convergent, has a limit w which satisfies the inequality

$$
\left|w-\frac{\bar{a}}{a \bar{a}-b^{2}}\right| \leqq \frac{b}{a \bar{a}-b^{2}},
$$

where

$$
\begin{aligned}
& a=1+a_{2}\left\{1-\frac{a_{3}}{1+a_{3}+a_{4}} \cdot \frac{\left|1+a_{3}+a_{4}\right|^{2}}{\left|1+a_{3}+a_{4}\right|^{2}-\left|a_{4}\right|^{2}}\right\} \\
& b=\frac{\left|a_{2} a_{3} a_{4}\right|}{\left|1+a_{3}+a_{4}\right|^{2}-\left|a_{4}\right|^{2}} .
\end{aligned}
$$

Put $a_{n}=u_{n}+i v_{n}$. Since, by hypothesis, $\left|a_{n}\right|-u_{n} \leqq \frac{1}{2}$, we may write $\left|a_{n}\right|=u_{n}+h_{n} / 2$, where $0 \leqq h_{n} \leqq 1$. Hence

$$
\begin{aligned}
\left|1+a_{2}\right| & \geqq 1+u_{2} \geqq u_{2}+\left(1+h_{2}\right) / 2=\left|a_{2}\right|+\frac{1}{2}, \\
\left|1+a_{2}+a_{3}\right| & \geqq 1+u_{2}+u_{3} \\
& =\left(1-h_{2} / 2-h_{3} / 2\right)+\left|a_{2}\right|+\left|a_{3}\right|>\left|a_{3}\right|, \\
\left|1+a_{n}+a_{n+1}\right| & \geqq 1+u_{n}+u_{n+1} \geqq u_{n}+h_{n} / 2+h_{n+1}+h_{n+1} / 2 \\
& \geqq\left|a_{n}\right|+\left|a_{n+1}\right|, \quad n=3,4,5, \cdots .
\end{aligned}
$$

If then we put

$$
g_{n}=\frac{\left|1+a_{2 n+1}+a_{2 n+2}\right|-\left|a_{2 n+2}\right|}{\left|1+a_{2 n+1}+a_{2 n+2}\right|}, \quad n=1,2,3,4, \cdots,
$$


we see that $0<g_{n} \leqq 1,(n=1,2,3, \cdots)$; and that

$$
\begin{aligned}
\left(1-g_{n-1}\right) g_{n} & \geqq \frac{\left|a_{2 n} a_{2 n+1}\right|}{\left|\left(1+a_{2 n-1}+a_{2 n}\right)\left(1+a_{2 n+1}+a_{2 n+2}\right)\right|}, \\
g_{1} & \geqq\left|a_{3}\right| /\left|1+a_{3}+a_{4}\right| .
\end{aligned}
$$

Define $x_{1}, x_{2}, x_{3}, \cdots$ by the equations

$$
\begin{gathered}
\frac{-a_{2} a_{3}}{\left(1+a_{2}\right)\left(1+a_{3}+a_{4}\right)}=g_{1} x_{1}, \\
\frac{-a_{2 n} a_{2 n+1}}{\left(1+a_{2 n-1}+a_{2 n}\right)\left(1+a_{2 n+1}+a_{2 n+2}\right)}=\left(1-g_{n-1}\right) g_{n} x_{n}
\end{gathered}
$$

$(n=2,3,4, \cdots)$, so that $\left|x_{1}\right| \leqq\left|a_{2}\right| /\left|1+a_{2}\right| \leqq 1-\left(1 / 2\left|1+a_{2}\right|\right)<1$; $\left|x_{n}\right| \leqq 1,(n=2,3,4, \cdots)$.

Now the even part ${ }^{3}$ of $(1.2)$ is

$$
\frac{1}{1+a_{2}}-\frac{a_{2} a_{3}}{1+a_{3}+a_{4}}-\frac{a_{4} a_{5}}{1+a_{5}+a_{6}}-\cdots \text {. }
$$

On making an equivalence transformation and introducing the expressions in (4.2) we find that this takes the form

$$
\frac{1}{1+a_{2}}\left\{\frac{1}{1}+\frac{g_{1} x_{1}}{1}+\frac{\left(1-g_{1}\right) g_{2} x_{2}}{1}+\ldots\right\} \text {. }
$$

In case some $a_{n}$ vanishes, the continued fraction terminates.

Now put

$$
z=\frac{g_{1}}{1}+\frac{\left(1-g_{1}\right) g_{2} x_{2}}{1}+\frac{\left(1-g_{2}\right) g_{3} x_{3}}{1}+\cdots,
$$

so that $w=1 /\left(1+a_{2}\right)\left(1+x_{1} z\right)$. For $z$ we have the inequality ${ }^{4}$

and consequently

$$
\left|z-\frac{1}{2-g_{1}}\right| \leqq \frac{1-g_{1}}{2-g_{1}}
$$

$$
\left|\frac{1}{w}-a\right| \leqq b,
$$

${ }^{3}$ CT, p. 160.

${ }^{4} \mathrm{H}$. S. Wall, $A$ class of functions bounded in the unit circle, Duke Mathematical Journal, vol. 7 (1940), pp. 146-153, p. 148. 
where $a$ and $b$ are as defined in the statement of the theorem. From this (4.1) now follows, and the theorem is proved.

Examples. If $a_{2}=2+3 i / 2, a_{3}=-i / 2, a_{4}=-1 / 4$ we find that

$$
\left|w-\frac{24(78-53 i)}{8893}\right| \leqq \frac{240}{8893} \text {. }
$$

If $a_{2}=1 / 2, a_{3}=1 / 6, a_{4}=1 / 3$, we find that

$$
|w-.69375| \leqq .00625 \text {. }
$$

In this case the first four partial quotients are the same as those in a continued fraction for $\log 2=.6931 \cdots$.

NORTHWESTERN UNIVERSITY

\section{SUR LE NOMBRE COMPLEXE BINAIRE}

\section{PEDRO F. CAPELLI}

\section{INTRODUCTION}

L'objet de ce travail est de démontrer que la théorie des fonctions polygènes ${ }^{1} d^{\prime}$ 'un variable complexe connu et celles d'un variable complexe duel et hyperbolique développées par les docteurs Vignaux et Durañona y $\mathrm{Vedia}^{2}$ sont des cas particuliers d'une même théorie qui en contient d'autres.

On doit l'origine de cette théorie à la suivante interprétation géométrique de la définition d'unité imaginaire $i^{2}=-1, j^{2}=1, k^{2}=0$.

Si nous considérons le point représentatif de cette unité, nous observons que son carré représente un autre point qui géométriquement signifie, dans le champ complexe ordinaire, une rotation de $+\pi / 2$, et, dans le champ complexe hyperbolique, une rotation de $-\pi / 2$ et dans le duel une translation à zéro.

Il n'y a rien de plus naturel que de considérer ces questions comme cas particuliers d'une rotation et d'une translation combinées, c'est-àdire que nous mettons un complexe $a+b \alpha$ dont l'unité imaginaire $\alpha$ est telle que $\alpha^{2}=\mu+\nu \alpha$, ce qui analytiquement exprime le concept géométrique que nous venons de dire. Quand

\footnotetext{
${ }^{1}$ Le mot polygène était introduit par E. Kasner. Voyez son premier papier: $A$ new theory of polygenic or nonmonogenic functions, Science, vol. 66 (1927), pp. 581-582.

${ }^{2}$ Sobre las funciones de una variable compleja hiperbólica, Contribución al Estudio de las Ciencias Físicomatemáticas, vol. 1, estudio $2^{\text {a }}$, 1935. J. C. Vignaux, Sobre la teoria de fonciones poligenas de una y varias variables complejas duales, ibid., estudio $3^{\mathrm{a}}$.
} 